\title{
Wireless ATM Layouts for Chain Networks ${ }^{*, * *}$
}

\author{
MICHELE FLAMMINI \\ Dipartimento di Informatica, University of L'Aquila, Via Vetoio loc. Coppito, I-67100 L'Aquila, Italy \\ GIORGIO GAMBOSI \\ Dipartimento di Matematica, University of Rome "Tor Vergata", Via della Ricerca Scientifica, I-00133 Rome, Italy
}

ALFREDO NAVARRA ***

Dipartimento di Informatica, University of L'Aquila, Via Vetoio loc. Coppito, I-67100 L'Aquila, Italy, and

MASCOTTE project, I3S-CNRS, INRIA, Université de Nice, Sophia Antipolis, route des Lucioles, B.P. 93 F-06902, Sophia Antipolis Cedex, France

\begin{abstract}
In this paper we consider the problem of constructing ATM layouts for wireless networks in which mobile users can move along a chain of base stations. We first show that deciding the existence of a layout with maximum hop count $h$, load $l$ and channel distance $d$ is NP-complete for every fixed value of $d$ greater or equal to 1 . We then provide optimal layout constructions for the case $d \leqslant 2$. Finally, optimal layout constructions are obtained also for any $d$ within the class of the so-called canonic layouts, that so far have always been shown to be the optimal ones.
\end{abstract}

Keywords: capacity planning, ATM networks, wireless networks, mobile users, chains

\section{Introduction}

The Asynchronous Transfer Mode (ATM for short) is the most popular networking paradigm for Broadband ISDN $[18,19,24]$. It transfers data in the form of small fixed-size cells, and in order to achieve the stringent transfer rate requirements, is based on two types of predetermined routes in the network: virtual paths or VPs, constituted by a sequence of successive edges or physical links, and virtual channels or $\mathrm{VCs}$, each given by the concatenation of a proper sequence of VPs. Routing in virtual paths can be performed very efficiently by dedicated hardware, while a cell passing from one virtual path to another one requires more complex and slower elaboration.

Given a network and a set of connections to be established, to provide the performance required by B-ISDN applications it is important that routing is performed in a hardware fashion in most of the nodes a cell traverses, at the same time limiting the number of paths sharing a same physical link $[1,4,15,25,26]$.

A graph theoretical model related to this ATM design problem has been first proposed in $[7,15]$. In such a framework, the VP layouts determined by the VPs constructed on the network are evaluated mainly with respect to two different cost measures: the hop count, that is the maximum number of

\footnotetext{
* Work supported by the IST Programme of the EU under contract number IST-1999-14186 (ALCOM-FT), by the EU RTN project ARACNE by the Italian project REAL-WINE, partially funded by the Italian Ministry of Education, University and Research, by the French MASCOTTE project I3S-CNRS/INRIA/Univ. Nice, Sophia Antipolis and by the Italian CNR project CNRG003EF8 - "Algoritmi per Wireless Networks" (AL-WINE).

** Preliminary version of this paper appeared in [11].

*** Corresponding author.
}

VPs belonging to a VC, which represents the number of VP changes of messages along their route to the destination, and the load, given by the maximum number of virtual paths sharing an edge, that determines the size of the VP routing tables (see, e.g., [8]). For further details and technical justifications of the model for ATM networks see for instance [1,15].

While the problem of determining VP layouts with bounded hop count and load is NP-hard under different assumptions $[10,15]$, many optimal and near optimal constructions have been given for various interconnection networks such as chain, trees, grids and so forth $[3,7,9,13,14,21,29]$ (see [30] for a survey).

The integration of wireless and ATM networks is emerging as one of the most promising approaches able to support users mobility while maintaining the quality of service offered by the classical ATM. This combination occurs at different levels and yields different scenarios, such as End-to-End WATM and WATM Interworking, applied respectively to create new wireless networks with ATM virtual channels extending until the mobile terminals and at a more external level for interconnecting different existing wireless subnets [16]. In both scenarios, the mobility facility requires the efficient solution of several problems, such as handover (users movement), routing, location management, connection control and so forth. A detailed discussion of these and other related issues can be found in $[2,5,6,16,23,27]$.

An extension of the basic ATM model of [7,15] able to combine quality of service and mobility aspects in wireless ATM networks has been proposed in [12]. In this model a subset of the nodes of the network represents the base stations and users are allowed to move between them according to an adjacency graph expressing their adjacencies in the geographic space. Such a graph, in general, can differ from 
the physical topology of the infrastructured network. For instance, in nowadays cellular systems like GSM, the physical graph $G$ is a tree, stations correspond to its leaves and the adjacency graph is an hexagonal grid (see, for instance, [22]). Standard ATM layouts must be constructed in order to establish a different VC for each station, but their performance is evaluated by means of a further parameter, the virtual channel distance, that measures the time needed to reconstruct virtual channels during handover phases, that is when mobile terminals switch between adjacent stations. More precisely, the distance between the virtual channels of two adjacent nodes is equal to the number of VPs that must be deleted and added to one $\mathrm{VC}$ in order to obtain the other one. In order to make the rerouting phase imperceptible to users and thus to obtain a sufficient quality of service, the maximum distance between two virtual channels must be maintained as low as possible. Therefore, a natural combinatorial problem arises in which suitable trade-offs must be determined between the different performance measures.

In [12] it has been shown that the layout construction problem is intractable, that is NP-hard. Moreover, optimal layout constructions are given when the physical and adjacency graphs are coincident and correspond to basic interconnection networks, such as chains and rings. Such results hold under the assumption that all the VCs induce shortest paths in the underlying network.

In this paper we consider the determination of WATM layouts for chains in the non-shortest path case in which the lengths of the paths induced by the VPs is not constrained. We first show that deciding the existence of a layout with maximum hop count $h$, load $l=1$ and distance $d=1$ is NP-complete even when the adjacency graph is a chain of base stations with the source coinciding with one of its endpoints. Moreover, such a hardness result is extended to every fixed value of $d$. We then consider the case in which the physical and adjacency graph coincide with chains and provide optimal layout constructions for $d \leqslant 2$. Finally, optimal layout constructions are obtained also for any $d$ within the class of the so-called canonic layouts, that so far have been always shown to be the optimal ones.

The paper is organized as follows. In the next section we introduce the model, the notation and the necessary definitions. In section 3 we provide the above mentioned hardness results for the layout construction problem. In section 4 we provide the optimal layouts for chains when $d=2$ and in section 5 the optimal canonic ones for any $d$. Finally, in section 6 , we give some conclusive remarks and discuss some open questions.

\section{The WATM model}

We model the network as an undirected graph $G=(V, E)$, where nodes in $V$ represent switches and edges in $E$ are pointto-point communication links. In $G$ there exists a subset of nodes $U \subseteq V$ constituted by base stations, i.e., switches adapted to support mobility and having the additional capa- bility of establishing wireless connections with mobile terminals. A distinguished source node $s \in V$ provides high speed services to the users moving along the network. We observe that, according to the wireless nature of the system, during the handover phase mobile terminals do not necessarily have to move along the network $G$, but they can switch directly from one station to another, provided that they are adjacent in the physical space. It is thus possible to define a (connected) adjacency graph $A=(U, F)$, whose edges in $F$ represent adjacencies between stations.

A layout $\Psi$ for $G=(V, E)$ with source $s \in V$ is a collection of simple paths in $G$, termed virtual paths (VPs for short), and a mapping that defines, for each station $u \in U$, a unique virtual channel $V C(u)$ connecting $s$ to $u$, i.e., a simple path from $s$ to $u$ in the virtual topology defined by the VPs of $\Psi$. In other words, $V C(u)$ is a collection of VPs whose concatenation forms a path in $G$ from $s$ to $u$.

Definition 2.1 [15]. The hop count $h(u)$ of a node $u \in U$ in a layout $\Psi$ is the number of VPs contained in $V C(u)$, that is $|V C(u)|$. The maximal hop count of $\Psi$ is $\mathcal{H}_{\max }(\Psi) \equiv$ $\max _{u \in U}\{h(u)\}$.

Definition 2.2 [15]. The load $l(e)$ of an edge $e \in E$ in a layout $\Psi$ is the number of VPs $\psi \in \Psi$ that include $e$. The maximal load $\mathcal{L}_{\max }(\Psi)$ of $\Psi$ is $\max _{e \in E}\{l(e)\}$.

As already observed, when passing from a station $u \in U$ to an adjacent one $v \in U$, the virtual channel $V C(v)$ must be reconstructed from $V C(u)$ changing only a limited number of VPs. Once fixed $V C(u)$ and $V C(v)$, denoted as $V C(u, v)$ the set of VPs in the subchannel corresponding to the longest common prefix of $V C(u)$ and $V C(v)$, this requires the deletion of all the VPs of $V C(u)$ that occur after $V C(u, v)$, plus the addition of all the VPs of $V C(v)$ after $V C(u, v)$. The number of removed and added VPs, denoted as $D(V C(u), V C(v))$, is called the distance of $V C(u)$ and $V C(v)$ and naturally defines a channel distance measure $d$ between pairs of adjacent nodes in $A$.

Definition 2.3 [12]. The channel distance of two nodes $u$ and $v$, such that, $\{u, v\} \in F$ (i.e., adjacent in $A$ ) is $d(u, v)=$ $D(V C(u), V C(v))=h(u)+h(v)-2|V C(u, v)|$. The maximal distance of $\Psi$ is $\mathcal{D}_{\max }(\Psi) \equiv \max _{\{u, v\} \in F}\{d(u, v)\}$.

It is now possible to give the following definition concerning layouts for WATM networks.

Definition 2.4. A layout $\Psi$ with $\mathcal{H}_{\max }(\Psi) \leqslant h, \mathcal{L}_{\max }(\Psi)$ $\leqslant l$ and $\mathcal{D}_{\max }(\Psi) \leqslant d$ is a $\langle h, l, d\rangle$-layout for $G, s$ and $A$.

In the following we will always assume that all the VPs of $\Psi$ are contained in at least one VC. In fact, if such property does not hold, the not used VPs can be simply removed without increasing the performance measures $h, l$ and $d$.

Before concluding the section, let us remark that for practical purposes and quality of services guarantees, it makes 
sense to consider the case where $d \ll h$. In fact, while a little communication delay proportional to the hop count in general can be tolerated, connections gaps due to rerouting of virtual channels must not be appreciated by mobile users. On the other hand, when $d \geqslant 2 h$, our model coincides with the classical one presented in [15] for standard ATM networks, since the difference between any two virtual channels is always at most equal to $2 h$.

\section{Hardness and approximation results}

In this section we show that constructing optimal dynamic layouts is in general an NP-hard problem, even when $l=1$, $d=1$ and the adjacency graph is a chain of stations with the source being one of its endpoints.

Before proving our results, let us briefly outline the basic characteristics of a layout with maximum delay $d=1$.

Given any two stations $u_{1}, u_{2} \in U$ adjacent in $A=$ $(U, F)$, during an handover from $u_{1}$ to $u_{2}$ if $d=1$ by definition only one VP can be modified. This means that either $V C\left(u_{2}\right)$ is a prefix of $V C\left(u_{2}\right)$ and thus $V C\left(u_{2}\right)$ is obtained from $V C\left(u_{1}\right)$ adding a new VP from $u_{1}$ to $u_{2}$, or vice versa. In any case, a VP between $u_{1}$ and $u_{2}$ must be contained in the layout. As a direct consequence, the virtual topology defined by the VPs of $\Psi$ coincides with the adjacency graph $A$. Moreover, $A$ must be acyclic. In fact, moving from a station in one direction along a cycle it is not possible to rebuild the virtual channel of the station itself when it is reached twice. Finally, if the source coincides with a base station, the maximum hop count of $\Psi$ is the eccentricity of $s$ in $A$, that is, the maximum distance in $A$ between $s$ and the other stations.

We are now ready to prove our first hardness result.

Theorem 3.1. Given a network $G=(V, E)$, a source $s \in V$, a chain adjacency graph $A=(U, F)$ and a positive integer $h$, deciding the existence of a $\langle h, 1,1\rangle$-layout for $G$ with source $s$ is an NP-complete problem.

Proof. First of all, observe that, for any $h, l, d$, the problem of deciding the existence of a $\langle h, l, d\rangle$-layout is in NP, as given $G=(V, E), s \in V, A=(U, F)$ and a layout $\Psi$, it is possible to check in polynomial time whether $\mathcal{H}_{\max }(\Psi) \leqslant h$, $\mathcal{L}_{\max }(\Psi) \leqslant l$ and $\mathcal{D}_{\max }(\Psi) \leqslant d$.

We prove the claim by providing a polynomial time reduction from Disjoint Paths problem (DP), known to be NP-complete [20]. An instance of this problem is constituted by a graph $G=(V, E)$ and a collection of node pairs $\left\{\left(s_{1}, t_{1}\right), \ldots,\left(s_{k}, t_{k}\right)\right\}$. We want to determine whether there exist $k$ edge-disjoint paths in $G$, each connecting a different pair $\left(s_{1}, t_{1}\right), 1 \leqslant i \leqslant k$.

Without loss of generality, it is possible to assume that all the pairs $\left(s_{i}, t_{i}\right), 1 \leqslant i \leqslant k$, are disjoint, i.e., all nodes $s_{1}, \ldots, s_{k}, t_{1}, \ldots, t_{k}$ are different. In fact, any instance not satisfying this property can be trivially modified into an equivalent one in which every node $v$ occurring in $k^{\prime} \leqslant k$ pairs is connected in $G$ to $k^{\prime}$ new nodes $v_{1}, \ldots, v_{k^{\prime}}$ and the $k^{\prime}$ pairs contain in the order $v_{1}, \ldots, v_{k^{\prime}}$ instead of $v$.
Starting from an instance of DP, we construct a network $G^{\prime}=\left(V^{\prime}, E^{\prime}\right)$, a source $s \in V^{\prime}$ and a chain adjacency graph $A=(U, F)$ that admit a $\langle h, 1,1\rangle$-layout with $h=2 k-1$ if and only if there exist the requested $k$ edge-disjoint paths in the instance of DP.

Let $G^{\prime}=\left(V^{\prime}, E^{\prime}\right)$ be such that, given $k-1$ nodes $w_{1}, \ldots, w_{k-1}$ not contained in the initial graph $G, V^{\prime}=V \cup$ $\left\{w_{1}, \ldots, w_{k-1}\right\}$ and $E^{\prime}=E \cup\left\{\left\{t_{i}, w_{i}\right\}\left\{w_{i}, s_{i+1}\right\} \mid 1 \leqslant i<k\right\}$. Concerning $A=(U, F)$, let $U=\left\{s_{1}, \ldots, s_{k}, t_{1}, \ldots, t_{k}\right\}$ and $F=\left\{\left\{s_{i}, t_{i}\right\} \mid 1 \leqslant i \leqslant k\right\} \cup\left\{\left\{t_{i}, s_{i+1}\right\} \mid 1 \leqslant i<k\right\}$. Finally, the source $s=s_{1}$.

Assume first that there is a $\langle 2 k-1,1,1\rangle$-layout $\Psi$ for $G^{\prime}=\left(V^{\prime}, E^{\prime}\right), s$ and $A=(U, F)$. By the considerations at the beginning of this section, for each $e \in F$, a VP in $\Psi$ must exist connecting the two endpoints of $e$. We can assume that for each $i, 1 \leqslant i<k$, the VP connecting $t_{i}$ to $s_{i+1}$ is $\left\langle t_{i}, w_{i}, s_{i+1}\right\rangle$, i.e., it is constituted by the new added path in $G^{\prime}$ that goes from $t_{i}$ to $s_{i+1}$ through the new node $w_{i}$. In fact, if this does not hold, it is possible to add to $\Psi$ the new $\mathrm{VP}\left\langle t_{i}, w_{i}, s_{i+1}\right\rangle$, deleting the old one and then, in order to keep $l=1$, if there is another VP stepping through $w_{i}$, it is modified in such a way that its subpath between $t_{i}$ and $s_{i+1}$ coincides with the old deleted VP.

Therefore, since $l=1$ and for all $i, 1 \leqslant i \leqslant k$, the VP between $s_{i}$ and $t_{i}$ does not step through any of the nodes $w_{1}, \ldots, w_{k-1}$, there must exist $k$ edge-disjoint paths in $G$ connecting the pairs $\left(s_{1}, t_{1}\right), \ldots,\left(s_{k}, t_{k}\right)$.

Vice versa, if there are $k$ edge-disjoint paths in $G$ connecting the pairs $\left(s_{1}, t_{1}\right), \ldots,\left(s_{k}, t_{k}\right)$, a $\langle 2 k-1,1,1\rangle$-layout $\Psi$ for $G^{\prime}=\left(V^{\prime}, E^{\prime}\right), s$ and $A=(U, F)$ can be constructed as follows. For each $i, 1 \leqslant i \leqslant k$, the VP between $s_{i}$ and $t_{i}$ is given by the corresponding path in $G$, edge-disjoint with all the others. The VP between $t_{i}$ and $s_{i+1}, 1 \leqslant i<k$, is $\left\langle t_{i}, w_{i}, s_{i+1}\right\rangle$. Since $s=s_{1}$ and the eccentricity in $A=(U, F)$ of the station $s_{1}$ is $2 k-1$, the layout $\Psi$ thus constructed gives directly a $\langle h, 1,1\rangle$-layout with $h=2 k-1$.

Notice that in the above construction the source $s$ corresponds to an endpoint of the chain $A=(U, F)$, so as already remarked the NP-completeness holds also under this restriction.

The above result can generalized to any fixed $d>0$ as follows.

Theorem 3.2. For any fixed integer $d>0$, given a network $G=(V, E)$, a source $s \in V$, a chain adjacency graph $A=(U, F)$ and a positive integer $h$, deciding the existence of a $\langle h, 1, d\rangle$-layout for $G$ with source $s$ is an NP-complete problem.

Proof. Given $G$, a source $s$ and an adjacency graph $A=$ $(U, F)$, it is sufficient to construct in polynomial time $G^{\prime}, s^{\prime}$ and $A^{\prime}=\left(U^{\prime}, F^{\prime}\right)$, such that, $G, s, A$ admit a $\langle h, 1,1\rangle$-layout if and only if $G^{\prime}, s^{\prime}, A^{\prime}$ admit a $\left\langle h^{\prime}, 1, d\right\rangle$-layout for a suitable $h^{\prime}>0$.

By theorem 3.1, it is possible to assume that $A=(U, F)$ is a chain of the nodes $u_{1}, \ldots, u_{k}$ and $s=u_{1}$. 
$G^{\prime}=\left(V^{\prime}, E^{\prime}\right)$ is obtained from $G=(V, E)$ by adding for each $u_{i}, 1 \leqslant i<k, d-1$ other stations $u_{i, 1}, \ldots, u_{i, d-1}$ connected by edges $\left\{u_{i}, u_{i, 1}\right\}$ and $\left\{u_{i, j}, u_{i, j+1}\right\}, 1 \leqslant j<$ $d-1$, in such a way that $u_{i}, u_{i, 1}, \ldots, u_{i, d-1}$ form a chain of $d$ nodes. $s^{\prime}=u_{1}$ and the new adjacency graph $A^{\prime}=\left(U^{\prime}, F^{\prime}\right)$ is, such that, $U^{\prime}=U \cup\left\{u_{i, 1}, \ldots, u_{i, d-1} \mid 1 \leqslant i \leqslant k\right\}$ and $F^{\prime}=\left\{\left\{u_{i}, u_{i, 1} \mid 1 \leqslant i \leqslant d-1\right\} \cup\left\{\left\{u_{i, j}, u_{i, j+1} \mid 1 \leqslant i<k\right.\right.\right.$, $1 \leqslant j<d-1\} \cup\left\{\left\{u_{i, d-1}, u_{i+1} \mid 1 \leqslant i<k\right\}\right.$. Hence, $A=(U, F)$ is a chain.

Since $l=1$, in any layout $\Psi^{\prime}$ for $G^{\prime}, s^{\prime}, A^{\prime}$, all edges edges $\left\{u_{i}, u_{i, 1}\right\}$ and $\left\{u_{i, j}, u_{i, j+1}\right\}, 1 \leqslant i<k$ and $1 \leqslant j<d-1$, must be VPs, as they are the only simple paths connecting the respective endpoint stations. Then, during the handover from a station $u_{i, d-1}$ to $u_{i+1}$, the $d-1 \operatorname{VPs}\left\{u_{i}, u_{i, 1}\right\}$ and $\left\{u_{i, j}, u_{i, j+1}\right\}, 1 \leqslant j<d-1$, must be deleted and then a single VP must be added from $u_{i}$ (the last station in the common prefix of the virtual channels $V C\left(u_{i, d-1}\right)$ and $\left.V C\left(u_{i+1}\right)\right)$ to $u_{i+1}$.

Since $s^{\prime}=u_{1}$ and $u_{1}$ has eccentricity $h^{\prime}=\max \{h, k-2+$ $d-2\}$ in $A^{\prime}=\left(U^{\prime}, F^{\prime}\right)$, then $G, s, A$ admit a $\langle h, 1,1\rangle$-layout if and only if $G^{\prime}, s^{\prime}, A^{\prime}$ admit a $\left\langle h^{\prime}, 1, d\right\rangle$-layout, hence the theorem holds.

Again, the NP-completeness still holds if the source $s$ is an endpoint of the chain adjacency graph.

Before concluding the section, let us, finally, show that for $d=1$ a stronger hardness result holds. To this aim observe first that as remarked at the beginning of this section, the virtual topology induced by any $\langle h, l, 1\rangle$-layout coincides with the adjacency graph. Moreover, if the source coincides with a base station, $h$ is equal to the eccentricity in $A$ of $s$, otherwise connecting $s$ by a VP to a node of minimum eccentricity in $A$ it is possible to obtain a layout with a maximum hop count equal to its eccentricity increasing the load at most of one. Therefore, as far as approximation results are concerned, the interesting parameter to be approximated remains the maximum load.

The problem of minimizing the maximum load is equivalent from an approximation point of view to the optimization version of the decision problem DP in which we want to determine $k$ paths connecting the $k$ source-destination pairs $\left(s_{1}, t_{1}\right), \ldots,\left(s_{k}, t_{k}\right)$ in such a way as to minimize the maximum number of paths sharing a same edge.

In fact, any $r$-approximation algorithm $A$ for the layout problem yields directly a $\mathrm{O}(r)$-approximation algorithm $A_{\mathrm{DP}}$ for DP. Informally, $A_{\mathrm{DP}}$ simply consists in running $A$ on the instance of the layout problem obtained by adding a new source $s$, connecting $s$ to each $s_{i}, 1 \leqslant i \leqslant k$, and letting $A=(U, F)$ be, such that, $U=\left\{s, s_{1}, \ldots, s_{k}, t_{1}, \ldots, t_{k}\right\}$ and $F=\left\{\left\{s, s_{i}\right\} \mid 1 \leqslant i \leqslant k\right\} \cup\left\{\left\{s_{i}, t_{i}\right\} \mid 1 \leqslant i \leqslant k\right\}$. The $k$ VPs connecting each $s_{i}$ to $t_{i}, 1 \leqslant i \leqslant k$, in the layout returned by $A$ correspond to an $\mathrm{O}(r)$-approximate solution for DP. A reverse reduction can be determined by observing that an $r$-approximation algorithm $A_{\mathrm{DP}}$ for DP yields directly an $\mathrm{O}(r)$-approximation algorithm $A$ for the layout construction problem that consists in running $A_{\mathrm{DP}}$ on the instance obtained by associating a source-destination $(s, t)$ to each edge $\{s, t\} \in F$. The paths returned by $A_{\mathrm{DP}}$ plus an eventual path connecting $s$ to the node with minimum eccentricity in $A$ if $s$ is not a base station form the VPs of an $\mathrm{O}(r)$-approximate solution for the layout problem.

To the best of our knowledge, the best general algorithm for DP has an approximation ratio $r=\mathrm{O}(\sqrt{|E| \log |V|})$, while $r=\mathrm{O}($ polylog $|V|)$ [28]. Therefore, an $\mathrm{O}(\sqrt{|E| \log |V|})$ approximation algorithm exists for the maximum load minimization in layout with $d=1$, while any algorithm with an asymptotic better approximation ratio would improve upon [28].

\section{Optimal chain layouts for $d \leqslant 2$}

Starting from the hardness results shown in the previous section, we now focus on specific topologies and provide optimal layouts for chain networks when the maximum channel distance $d$ is at most 2. More precisely, we consider the case in which the physical graph is a chain $C_{n}$ of $n$ nodes, that is $V=\{1,2, \ldots, n\}, E=\{\{v, v+1\} \mid 1 \leqslant v \leqslant n-1\}$ and the adjacency graph $A$ coincides with $C_{n}$. Moreover, without loss of generality, we take the leftmost node of the chain as the source, i.e., $s=1$, as otherwise we can split the layout construction problem into two equivalent independent subproblems for the left- and the right-hand sides of the source, respectively.

Given fixed $h, l, d$ and a $\langle h, l, d\rangle$-layout $\Psi$ for a chain $C_{n}$, we say that $\Psi$ is optimal if no $\langle h, l, d\rangle$-layout exists for any chain $C_{m}$ with $m>n$.

By the considerations of the previous section for $d=1$, the virtual topology induced by the VPs of any $\langle h, l, 1\rangle$-layout $\Psi$ coincides with the adjacency graph $A$ and thus with $C_{n}$. As a consequence, the largest chain admitting a $\langle h, l, 1\rangle$-layout is, such that, $n=h+1$. Therefore, in the remaining part of this section we focus on the case $d=2$.

In the following we denote by $\langle u, v\rangle$ the unique VP corresponding to the simple path from $u$ to $v$ in $C_{n}$ and by $\left\langle\left\langle s, v_{1}\right\rangle\left\langle v_{1}, v_{2}\right\rangle \ldots\left\langle v_{k}, v\right\rangle\right\rangle$ or simply $\left\langle s, v_{1}, v_{2}, \ldots, v_{k}, v\right\rangle$ the virtual channel $V C(v)$ of $v$ given by the concatenation of the $\operatorname{VPs}\left\langle s, v_{1}\right\rangle,\left\langle v_{1}, v_{2}\right\rangle, \ldots,\left\langle v_{k}, v\right\rangle$.

The following lemma establishes that, when moving in one direction along a chain, some VPs are "accumulated", that is they cannot be removed from the VCs of the successive nodes encountered along the same direction.

Lemma 4.1. Given a $\langle h, l, 2\rangle$-layout $\Psi$ for a chain network and a node $v$, if $V C(v)=\left\langle s, v_{1}, v_{2}, \ldots, v_{k}, v\right\rangle$ and in $V C(v)$ there exist two consecutive VPs $\left\langle v_{i-1}, v_{i}\right\rangle,\left\langle v_{i}, v_{i+1}\right\rangle$ with $v_{i-1}<v$ and $v_{i}<v$ (resp. $v_{i-1}>v$ and $v_{i}>v$ ), then for every $u \geqslant v$ (resp. $u \leqslant v),\left\langle s, v_{1}, v_{2}, \ldots, v_{i-1}, v_{i}\right\rangle$ is a prefix of $V C(u)$.

Proof. Assume first that $v_{i-1}<v$ and $v_{i}<v$ and let $u>v$ be the first node, such that, $\left\langle v_{i}, v_{i+1}\right\rangle \notin V C(u)$. Since $d(u-1, u) \leqslant 2$, to reach $u$ we can only add $\left\langle v_{i}, u\right\rangle$. This process can be iterated to every node $w$ with $w>u$, hence the 
claim holds. An analogous argument applies when $v_{i-1}>v$ and $v_{i}>v$.

Another useful property of $\langle h, l, 2\rangle$-layouts is that the prefixes of a VC are the VCs of their final nodes.

Lemma 4.2. There exists an optimal $\langle h, l, 2\rangle$-layout $\Psi$ for a chain network, such that, for every node $v$ with $V C(v)=$ $\left\langle s, v_{1}, v_{2}, \ldots, v_{k-1}, v_{k}, v\right\rangle, V C\left(v_{i}\right)=\left\langle s, v_{1}, v_{2}, \ldots, v_{i}\right\rangle$ for every $i \leqslant k$.

Proof. Let $\Psi$ be any optimal $\langle h, l, 2\rangle$-layout. We now prove that, if for a given node $v$ each prefix of every virtual channel $V C(u)$ with $u<v$ is the virtual channel of the corresponding final node, then $\Psi$ can be modified in such a way that such a property is satisfied also by $V C(v)$. This clearly proves the lemma.

Trivially the property is satisfied by the virtual channel of source $s$, since it is empty. Therefore, let $v \geqslant 2$ be any node, such that, the property is true for all the VCs of the previous nodes and let $V C(v)=\left\langle s, v_{1}, v_{2}, \ldots, v_{k-1}, v_{k}, v\right\rangle$. Recalling that $d(v-1, v) \leqslant 2$, it is possible to distinguish among the following cases:

1. $V C(v-1)=\left\langle s, v_{1}, v_{2}, \ldots, v_{k}, v-1\right\rangle$,

2. $V C(v-1)=\left\langle s, v_{1}, v_{2}, \ldots, v_{k}, v, v-1\right\rangle$,

3. $V C(v-1)=\left\langle s, v_{1}, v_{2}, \ldots, v_{k}\right\rangle$, that is $v_{k}=v-1$,

4. $V C(v-1)=\left\langle s, v_{1}, v_{2}, \ldots, v_{k}, v_{k+1}, v\right\rangle$, and

5. $V C(v-1)=\left\langle s, v_{1}, v_{2}, \ldots, v_{k-1}\right\rangle$, that is $v_{k-1}=v-1$.

Since by the hypothesis the claim is true for $V C(v-1)$ and $\left\langle s, v_{1}, v_{2}, \ldots, v_{k}\right\rangle$ is a prefix of $V C(v-1), V C\left(v_{i}\right)=$ $\left\langle s, v_{1}, v_{2}, \ldots, v_{i}\right\rangle$ for each $v_{i}$ with $1 \leqslant i \leqslant k$. Therefore, every prefix of $V C(v)$ is a VC.

If $V C(v-1)=\left\langle s, v_{1}, v_{2}, \ldots, v_{k-1}\right\rangle$, that is $v_{k-1}=v-1$, we further distinguish the following two subcases.

(I) $v_{k}>v$. In this case the $\mathrm{VP}\left\langle v, v_{k}\right\rangle$ must be deleted in the VC of a node $u$ with $v<u \leqslant v_{k}$. If $u=v_{k} V C\left(v_{k}\right)=\left\langle s, v_{1}, v_{2}, \ldots, v_{k}\right\rangle$, otherwise, since $d(u-1, u) \leqslant 2, V C(u)=\left\langle s, v_{1}, v_{2}, \ldots, v_{k}, u\right\rangle$ and iterating the same argument to the $\operatorname{VP}\left\langle u, v_{k}\right\rangle$ we finally have that again $V C\left(v_{k}\right)=\left\langle s, v_{1}, v_{2}, \ldots, v_{k}\right\rangle$. Therefore, since for each $v_{i}$ with $i<k\left\langle s, v_{1}, v_{2}, \ldots, v_{i}\right\rangle$ is a prefix of $V C(v-1)$, we have $V C\left(v_{i}\right)=\left\langle s, v_{1}, v_{2}, \ldots, v_{i}\right\rangle$ for every $v_{i}$ with $1 \leqslant i \leqslant k$.

(II) $v_{k}<v-1$. If the $\operatorname{VP}\left\langle v_{k}, v-1\right\rangle$ is contained in the $\mathrm{VC}$ of a node $u<v-1$, then starting from the source $s$, in $V C(u)\left\langle v_{k}, v-1\right\rangle$ is not traversed from $v_{k}$ to $v-1$, otherwise by hypothesis $\left\langle v_{k}, v-1\right\rangle$ would be contained in $V C(v-1)$ and thus it could not be added to $V C(v-1)$ with $\left\langle v_{k}, v\right\rangle$ to obtain $V C(v)$. Therefore, $\left\langle v_{k}, v-1\right\rangle$ is traversed from $v-1$ to $v_{k}$ and again by hypothesis the prefix of $V C(u)$ till $v-1$ coincides with $V C(v-1)$ and $V C\left(v_{k}\right)=\left\langle s, v_{1}, v_{2}, \ldots, v_{k-1}, v_{k}\right\rangle$. As in the previous subcase, since for each $v_{i}$ with $i<k$ $\left\langle s, v_{1}, v_{2}, \ldots, v_{i}\right\rangle$ is a prefix of $\operatorname{VC}(v-1)$, we have $V C\left(v_{i}\right)=$ $\left\langle s, v_{1}, v_{2}, \ldots, v_{i}\right\rangle$ for every $v_{i}$ with $1 \leqslant i \leqslant k$.
Assume then that the $\operatorname{VP}\left\langle v_{k}, v-1\right\rangle$ is not contained in $V C(u)$ for every $u<v-1$. In this case also $\left\langle v_{k}, v\right\rangle$ is not contained in any $V C(u)$ with $u<v-1$, because otherwise, with no matter of the sense in which $\left\langle v_{k}, v\right\rangle$ is traversed in $V C(u), V C(v)$ could not contain $\left\langle v_{k}, v-1\right\rangle$, as by hypotheses $\left\langle v_{k}, v-1\right\rangle$ does not belong to $V C(u)$.

If $\left\langle v_{k}, v\right\rangle$ is contained in $V C(v+1)$, then by lemma 4.1 $V C(v)$ is a prefix of all the $\operatorname{VCs} V C(u)$ with $u \geqslant v$. Therefore, the layout obtained by deleting the $\operatorname{VPs}\left\langle v_{k}, v-1\right\rangle$ and $\left\langle v_{k}, v\right\rangle$, adding $\langle v-1, v\rangle$ and modifying each $V C(u)=\left\langle s, v_{1}, v_{2}, \ldots\right.$, $\left.v-1, v_{k}, v, \ldots, u\right\rangle$ with $u \geqslant v$ as $V C(u)=\left\langle s, v_{1}, v_{2}, \ldots\right.$, $v-1, v, \ldots, u\rangle$ does not increase the hop count of any node, the load of any edge and the channel distance of the adjacent nodes. Therefore, since in the new layout the virtual channels of the nodes before $v$ are not modified and $V C(v)=\left\langle s, v_{1}, v_{2}, \ldots, v-1, v\right\rangle=\left\langle s, v_{1}, v_{2}, \ldots, v_{k-1}, v\right\rangle$, $\left\langle s, v_{1}, v_{2}, \ldots, v_{i}\right\rangle$ is a prefix of $V C(v-1)$ for every $v_{i}$ with $1 \leqslant i<k$ and thus $V C\left(v_{i}\right)=\left\langle s, v_{1}, v_{2}, \ldots, v_{i}\right\rangle$.

If $\left\langle v_{k}, v\right\rangle$ is not contained in $V C(v+1)$, then by lemma 4.1 $\left\langle s, v_{1}, v_{2}, \ldots, v-1, v_{k}\right\rangle$ is a prefix of all the VCs $V C(u)$ with $u \geqslant v$. Moreover, all the VPs starting at $v_{k}$ contained in the VC of some node $u>v$ are not contained in any VC $V C(w)$ with $w \leqslant v-1$, as otherwise by hypothesis $V C(u)$ would not contain $\left\langle v_{k}, v-1\right\rangle$. Notice also that the other endpoint of each such VP is greater than $v$, as otherwise by the maximum channel distance it cannot be used in the VCs of the nodes after $v$. It is thus possible to modify the layout $\Psi$ as follows. The VPs $\left\langle v_{k}, v-1\right\rangle$ and $\left\langle v_{k}, v\right\rangle$ are deleted, $\langle v-1, v\rangle$ is added, the VPs $\left\langle v_{k}, w\right\rangle$ contained in the VC of some node $u>v$ are substituted with $\langle v, w\rangle$ and, finally, each $V C(u)=\left\langle s, v_{1}, v_{2}, \ldots, v-1, v_{k}, w, \ldots, u\right\rangle$ with $u \geqslant v$ is modified as $V C(u)=\left\langle s, v_{1}, v_{2}, \ldots, v-1, v, w, \ldots, u\right\rangle$. Вy the above considerations, the new layout does not increase the hop count of any node, the load of any edge and the channel distance of the adjacent nodes. Moreover, it does not modify the virtual channels of the nodes before $v$. Therefore, again $V C(v)=\left\langle s, v_{1}, v_{2}, \ldots, v-1, v\right\rangle=\left\langle s, v_{1}, v_{2}, \ldots, v_{k-1}, v\right\rangle$, $\left\langle s, v_{1}, v_{2}, \ldots, v_{i}\right\rangle$ is a prefix of $V C(v-1)$ for every $v_{i}$ with $1 \leqslant i<k$ and thus $\operatorname{VC}\left(v_{i}\right)=\left\langle s, v_{1}, v_{2}, \ldots, v_{i}\right\rangle$.

Motivated by the previous lemma, even if not explicitly stated, in the remaining part of this section, we restrict our attention to layouts in which all the prefixes of each $\mathrm{VC}$ are the VCs of the corresponding final nodes. In fact, this does not affect the correctness of our results, since the optimality is preserved under such assumption.

The following corollary is a direct consequence of the previous lemma.

Corollary 4.3. Every VP of a $\langle h, l, 2\rangle$-layout $\Psi$ for a chain network is the final VP of exactly one of its two endpoints.

A last useful property that allows to suitably bound the maximum size of a chain admitting a $\langle h, l, 2\rangle$-layout is established in the following lemma.

Lemma 4.4. Given a $\langle h, l, 2\rangle$-layout $\Psi$ for a chain network and any $j \leqslant h$, let $u$ and $v$ be the last nodes with hop count 
$h(u)=j-1$ and $h(v)=j$, respectively. Then the last VPs of all the VCs reaching the nodes from $u+1$ to $v$ share a same physical link.

Proof. Let us first prove the claim for $j=1$, and thus with $u$ corresponding to the source $s=1$.

In order to show that the lemma holds for $j=1$, it is sufficient to prove that no two edge-disjoint VPs exist in the VCs of the nodes from $u+1$ to $v$.

Assume by contradiction that such property does not hold and let $\langle x, y\rangle,\langle w, z\rangle$ be a pair of closest edge-disjoint VPs in such VCs with $x<y \leqslant w<z \leqslant v$. Then $y=w$ or $y=w-1$, otherwise any other VP used to reach a node between $y$ and $w$ would be disjoint from $\langle x, y\rangle$ or $\langle w, z\rangle$ and closest to $\langle x, y\rangle$ or $\langle w, z\rangle$, thus contradicting the hypothesis.

If $y=w-1$ then $\langle x, y\rangle$ is not used to reach $y$. In fact, $\langle x, y\rangle$ cannot be contained in $V C(w)$, as by lemma 4.1 it would be contained also in $V C(v)$ against the hypothe$\operatorname{sis} h(v)=1$. Thus, if $\langle x, y\rangle$ is used to reach $y$, since $d(y, w) \leqslant 2$, when moving from $y$ to $w\langle x, y\rangle$ must be removed and the VP $\langle x, w\rangle$ must be added to reconstruct $V C(w)$. But then $\langle x, w\rangle$ would be a VP closer to $\langle w, z\rangle$ than $\langle x, y\rangle$, again contradicting the hypothesis.

By corollary $4.3,\langle x, y\rangle$ is then used to reach $x$. If $y$ is reached by a $\operatorname{VP}\langle q, y\rangle$ with $q<y$, then by corollary 4.3 $\langle w, z\rangle$ is used to reach $z$, and thus by lemma $4.1\langle q, y\rangle$ is contained in $V C(v)$ again contradicting the hypothesis $h(v)=1$. If $q>y\langle x, y\rangle$ and $\langle y, q\rangle$ would be closer than $\langle x, y\rangle$ and $\langle w, z\rangle$ : a contradiction.

Therefore, $y=w-1$ cannot hold and it must be $y=w$.

Recalling that by corollary 4.3 every VP is used to reach exactly one of its endpoints, i.e., it is the final VP of exactly one of the VCs of its endpoints, it is possible to distinguish the following cases:

- $\langle x, y\rangle$ reaches $x$ and $\langle y, z\rangle$ reaches $z$.

By corollary $4.3 y$ is reached by another $\operatorname{VP}\langle q, y\rangle$ with $q \neq x$ and $q \neq z$. If $q>y$, then by lemma $4.1\langle q, y\rangle$ is contained in all the VCs of the nodes before $x, x$ included, thus contradicting $h(s)=0$. Similarly, if $q<y,\langle q, y\rangle$ is contained in all the VCs of the nodes after $z, z$ included, thus contradicting $h(v)=1$. Therefore, this case cannot hold.

- $\langle x, y\rangle$ reaches $y$ and $\langle y, z\rangle$ reaches $z$.

Since by lemma 4.2 every prefix of $V C(z)$ corresponds to the $\mathrm{VC}$ of its final node, $\langle x, y\rangle$ is contained in $V C(z)$ and by lemma $4.1\langle x, y\rangle$ belongs to $V C(v)$, thus contradicting $h(v)=1$.

- $\langle x, y\rangle$ reaches $x$ and $\langle y, z\rangle$ reaches $y$.

This case is symmetric to the previous one and completely analogous considerations show that it would contradict $h(s)=0$.

- $\langle x, y\rangle$ reaches $y$ and $\langle y, z\rangle$ reaches $y$. This case is clearly impossible, since only one of the two VPs $\langle x, y\rangle$ and by $\langle y, z\rangle$ can be the last one of $V C(y)$.

In conclusion, neither $y=w$ can hold and therefore no two edge-disjoint VPs exist before $v$.

In order to extend the proof to every $j \leqslant h$, observe that since $u$ is the last node with hop count $h(u)=j-1$ and $d=2$, all the VCs of the nodes $w \geqslant u$ have $V C(u)$ as prefix. Thus an identical proof shows that all the VPs after $u$ in the VCs of the nodes $w$ with $u<w \leqslant v$ share a same physical edge.

It is thus possible to prove the following lemma.

Lemma 4.5. Given any $\langle h, l, 2\rangle$-layout $\Psi$ for a chain network and $j \leqslant h$, the last node $v$ with $h(v)=j$ is, such that, $v \leqslant j l+1$.

Proof. The claim trivially holds for $j=0$, since only the source $s=1$ has hop count $h(s)=0$.

Assume by induction that the corollary holds for a given $j$, such that, $1 \leqslant j<h$ and let $u \leqslant j l+1$ the last node with hop count $h(u)=j$ and $v$ the last node with hop count $h(v)=j+1$. Since by lemma 4.4 the last VPs of all the VCs reaching the nodes from $u+1$ to $v$ share a same physical link, there cannot be more than $l$ nodes from $u+1$ to $v$, otherwise the shared link would have load greater than $l$. Therefore, $v \leqslant u+l \leqslant(j+1) l+1$.

In conclusion, the following theorem holds.

Theorem 4.6. For every $h, l \geqslant 0$, a $\langle h, l, 2\rangle$-layout for a chain network $C_{n}$ is optimal if and only if $n=h l+1$.

Proof. By lemma 4.5, $n \leqslant h l+1$ holds for any $\langle h, l, 2\rangle$ layout for a chain $C_{n}$. A layout attaining $n=h l+1$ is depicted in figure 1 .

Before concluding the section, we finally observe that the layout in figure 1 is not the only optimal one. Another example with $h=4, l=4$ and $d=2$ is shown in figure 2 .

\section{Optimal canonic layouts for chain networks}

In this section we provide $\langle h, l, d\rangle$-layouts for chain networks that are optimal within the class of the canonic layouts. In

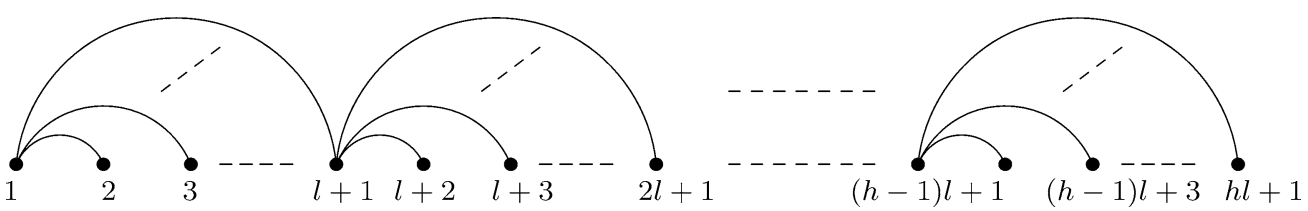

Figure 1. Optimal $\langle h, l, 2\rangle$-layout for a chain network 


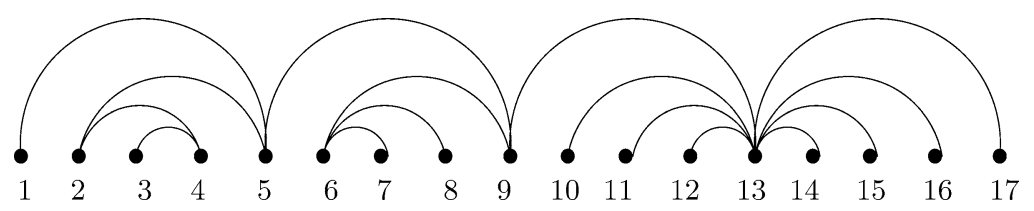

Figure 2. An alternative optimal $\langle 4,4,2\rangle$-layout.

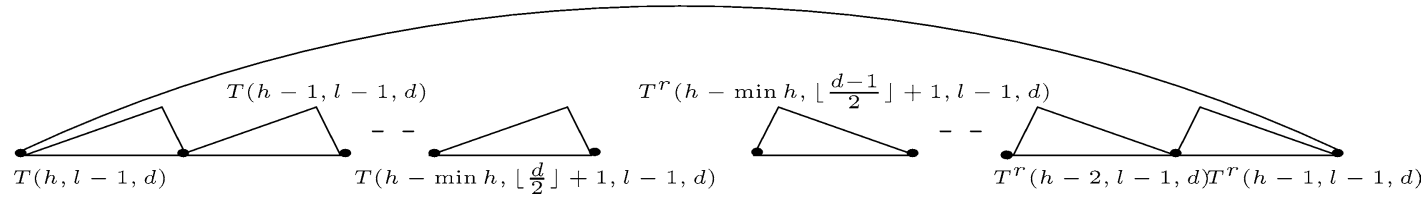

Figure 3. The recursive definition of $T(h, l, d)$ for $h>0$ and $l>0$.

fact, such layouts have been shown to be the optimal ones under different assumptions (see, for instance, $[12,15]$ ). Informally speaking, a layout $\Psi$ is canonic if it does not contain intersecting VPs and it induces a tree. More precisely, we have the following definitions.

Definition 5.1. Two VPs $\langle u, v\rangle$ and $\langle w, z\rangle$ are crossing if $u<$ $w<v<z$. A layout $\Psi$ is crossing-free if it does not contain any pair of crossing VPs.

Definition 5.2. A layout $\Psi$ is canonic if it is crossing-free and the virtual topology induced by its VPs is a tree.

Let us say that a rooted tree is ordered if a total order is defined on its nodes with the root being the lowest order node. Then there is a one-to-one corresponds between layouts for chains and ordered trees. Namely, each node of the tree corresponds to a node of the chain, the root corresponds to the source $s$, each edge to a VP of $\Psi$ and finally the total order of the nodes of the tree is given by the order of the nodes along the chain. Clearly, not all the ordered trees yield canonic layouts, as their induced VPs might be crossing. However, the one-to-one correspondence between ordered trees and canonic layouts is maintained if we restrict to ordered trees in which every subtree contains a subset of nodes that forms an interval according to the node ordering. In other words, each subtree corresponds to a segment of the chain not touched by the other subtrees.

Given any ordered tree $T$, let the reverse tree $T^{\mathrm{r}}$ be the symmetric ordered tree obtained from $T$ by inverting the order of the nodes (hence the root becomes the highest order node). We now introduce a new class of ordered trees $\Gamma(h, l, d)$ that allows to completely define the structure of an optimal $\langle h, l, d\rangle$-layout.

The definition of $\Gamma(h, l, d)$ is recursive and the solution of the associated recurrence gives the exact number of nodes reached by an optimal canonic $\langle h, l, d\rangle$-layout. Before introducing $\Gamma(h, l, d)$, let us define another ordered subtree that is exploited in its definition.

Definition 5.3. Given any $h, l, d, T(h, l, d)$ is an ordered tree recursively defined as follows.

- If $h=0$ or $l=0 T(h, l, d)$ consists of a single node.
- If $h>0$ and $l>0 T(h, l, d)$ contains at least two nodes and the lowest order node $u$, that is the root, is connected by an edge to the highest order node $v$.

Moreover, a chain of $\min \{h,\lfloor d / 2\rfloor\}$ trees $T(h-j, l-1, d)$ with $0 \leqslant j \leqslant \min \{h,\lfloor d / 2\rfloor\}-1$ is attached to $u$ in such a way that the lowest order node of $T(h, l-1, d)$ coincides with $u$ and the lowest order node of each $T(h-j, l-1, d)$ with $1 \leqslant j \leqslant \min \{h,\lfloor d / 2\rfloor\}-1$ coincides with the highest order node of $T(h-j+1, l-1, d)$.

Finally, a chain of $\min \{h-1,\lfloor(d-1) / 2\rfloor\}$ reverse trees $T^{\mathrm{r}}(h-j, l-1, d)$ with $1 \leqslant j \leqslant \min \{h-1,\lfloor(d-1) / 2\rfloor\}$ is attached to $v$ in such a way that the highest order node of $T^{\mathrm{r}}(h-1, l, d)$ coincides with $v$ and the highest order node of each $T^{\mathrm{r}}(h-j, l-1, d)$ with $2 \leqslant j \leqslant \min \{h-j$, $\lfloor(d-1) / 2\rfloor\}$ coincides with the lowest order node of $T^{\mathrm{r}}(h-j+1, l-1, d)$.

An example of $T(h, l, d)$ is depicted in figure 3. Informally speaking, a $T(h, l, d)$ corresponds to the sublayout of a canonic layout $\Psi$ induced by all the VPs occurring under a given VP, with the lowest order node being closer to the source. Thus, $T(h, l, d)$ is the subtree induced by all the VPs whose endpoints occur from the first endpoint of the given VP until the second endpoint.

Directly from the definition, it follows that all the nodes in $T(h, l, d)$ are at distance at most $h$ from $u$, and thus at most $h$ additional hops from the node corresponding to $u$ in $\Psi$ are sufficient to reach the other nodes corresponding to $T(h, l, d)$ in the chain. Moreover, the load yielded by $T(h, l, d)$ on its segment of the chain is bounded by $l$. Finally, the channel distance between two consecutive nodes belonging to the subchain of $T(h, l, d)$ is always at most equal to $d$. In fact, it is given by the maximum distance in $T(h, l, d)$ between two nodes adjacent in the ordering. Therefore, assuming by induction that such property holds inside the subtrees $T(j, l-1, d)$ (and thus $T^{\mathrm{r}}(j, l-1, d)$ ), in order to show that it holds also in $T(h, l, d)$ it is sufficient to prove that the final node of the chain of subtrees attached to $u$ and the final node of the other reverse chain attached to $v$, that is the only not yet considered adjacent pair of nodes, is at distance at most $d$. But such nodes are at distance $\min \{h,\lfloor d / 2\rfloor\}+1+\min \{h-1,\lfloor(d-1) / 2\rfloor\} \leqslant$ $\lfloor d / 2\rfloor+1+\lfloor(d-1) / 2\rfloor=d$. Therefore, also the channel 


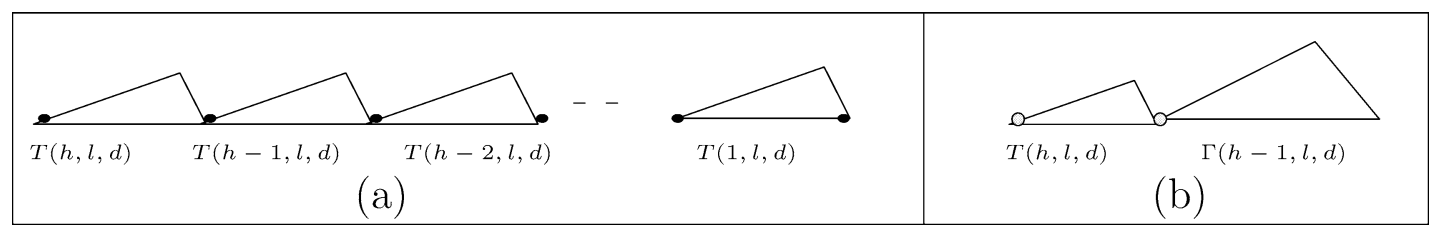

Figure 4. $\Gamma(h, l, d)$ in terms of trees of type $T$ (a) and the alternative recursive definition (b).

distance within the subchain of $T(h, l, d)$ is bounded by $d$. Clearly, symmetric considerations hold for each $T^{\mathrm{r}}(h, l, d)$.

We are now ready to define the final tree $\Gamma(h, l, d)$.

Definition 5.4. The ordered tree $\Gamma(h, l, d)$ is formed by the chain of $h$ trees $T(j, l, d), 1 \leqslant j \leqslant h$, such that, the lowest order node of $T(j, l, d)$ coincides the highest order node of $T(j+1, l, d)$ for $1 \leqslant j<h$ (see figure 4 ).

Notice that, if $h=0$ or $l=0, \Gamma(h, l, d)$ consists of just a single node. Moreover, an alternative recursive definition of $\Gamma(h, l, d)$ is given by a $T(h, l, d)$ attached to a $\Gamma(h-1, l, d)$ tree (again see figure 4 ).

Let $T_{n}(h, l, d)$ denote the number of nodes of $T(h, l, d)$ (and thus of $\left.T^{\mathrm{r}}(h, l, d)\right)$ minus one. Then, directly from definition $5.3, T_{n}(h, l, d)=0$ if $h=0$ or $l=0$, otherwise $T_{n}(h, l, d)=1+\sum_{j=0}^{\min \{h,\lfloor d / 2\rfloor\}-1} T_{n}(h-j, l-1, d)+$ $\sum_{j=1}^{\min \{h-1,\lfloor(d-1) / 2\rfloor\}} T_{n}(h-j, l-1, d)$.

Moreover, by definition 5.4 , denoted as $\Gamma_{n}(h, l, d)$ the number of nodes in $\Gamma(h, l, d), \quad \Gamma_{n}(h, l, d)=1+$ $\sum_{k=1}^{h} T_{n}(k, l, d)$.

Clearly, by the above observations, $\Gamma(h, l, d)$ corresponds to a canonic $\langle h, l, d\rangle$-layout for a chain network. Actually, a stronger result holds.

Lemma 5.5. The layout induced by $\Gamma(h, l, d)$ is optimal within the class of the canonic $\langle h, l, d\rangle$-layouts for chain networks.

Proof. Let $\Psi$ be any canonic $\langle h, l, d\rangle$-layout for a chain $C_{n}$. It is sufficient to show that $n \leqslant \Gamma_{n}(h, l, d)$.

Let $V C(n)=\left\langle v_{1}, \ldots, v_{k}\right\rangle$ with $v_{1}=s, v_{k}=n$ and $k \leqslant$ $h+1$ the VC of the last node of the chain in $\Psi$. We prove that $v_{i}-v_{i-1} \leqslant T_{n}(h-i+2, l, d)$ for every $i$, such that, $2 \leqslant i \leqslant k$. In fact, this implies $n=v_{k}=v_{1}+\sum_{i=2}^{k}\left(v_{i}-\right.$ $\left.v_{i-1}\right) \leqslant 1+\sum_{i=2}^{k} T_{n}(h-i+2, l, d)=1+\sum_{i=0}^{k-2} T_{n}(h-$ $i, l, d) \leqslant 1+\sum_{i=0}^{h-1} T_{n}(h-i, l, d)=1+\sum_{i=1}^{h} T_{n}(i, l, d)=$ $\Gamma_{n}(h, l, d)$.

In order to show that $v_{i}-v_{i-1} \leqslant T_{n}(h-i+2, l, d)$ for every $i$, such that, $2 \leqslant i \leqslant k$, it suffices to prove that, given any $\operatorname{VP}\langle u, v\rangle$ of a canonic $\langle h, l, d\rangle$-layout, such that, $h(u)=$ $h-h^{\prime}, h(v)=h-h^{\prime}+1$ or vice versa and there exist $l-l^{\prime}$ VPs over it, that is of the form $\langle w, z\rangle$ with $w \leqslant u$ and $z>v$ or $w<u$ and $z \geqslant v$, it is $v-u \leqslant T_{n}\left(h^{\prime}, l^{\prime}, d\right)$. If $l^{\prime}=1$, it must be $v=u+1$, otherwise the nodes between $u$ and $v$ could not be reached from the source without exceeding the maximum load $l$. Recalling definition 5.3, $v-u=1=$ $T_{n}\left(h^{\prime}, 1, d\right)$.
Assume then that the claim is true for $l^{\prime}-1$, that is, for all the VPs of a canonic $\langle h, l, d\rangle$-layout $\Psi$ with $l-l^{\prime}+1$ VPs over them and let $\langle u, v\rangle$ be a VP of $\Psi$ with $h(u)=h-h^{\prime}$, $h(v)=h-h^{\prime}+1$ and $l-l^{\prime}$ VPs over it. Let $w$ be the last node with $u \leqslant w \leqslant v$ (that is under $\langle u, v\rangle$ ) reached by a VC stepping through $u$ and not from $v$, and consider the subchain of $d_{1}$ VPs $\left\langle w_{1}, \ldots, w_{d_{1}+1}\right\rangle$ with $w_{1}=u$ and $w_{d_{1}+1}=w$ connecting $u$ to $w$. Similarly, let $\left\langle z_{d_{2}+1}, \ldots, z_{1}\right\rangle$ with $z_{1}=w+1$ and $z_{d_{2}+1}=v$ the subchain of $d_{2}$ VPs connecting $v$ to $w+1$. Since $w$ and $w+1$ are adjacent and the maximum channel distance is $d$, it must be $d_{1}+d_{2}+1 \leqslant d$. Moreover, since $h(w) \leqslant h$ and $h(w+1) \leqslant h, d_{1} \leqslant h^{\prime}$ and $d_{2} \leqslant h^{\prime}-1$. Therefore, since such subchains and with all their VPs occur under $\langle u, v\rangle$, by applying the inductive assumption it follows that

$$
\begin{aligned}
v-u= & (w-u)+(v-w) \\
= & \sum_{i=2}^{d_{1}+1}\left(w_{i}-w_{i-1}\right)+\left(1+\sum_{i=2}^{d_{2}+1}\left(z_{i}-z_{i-1}\right)\right) \\
\leqslant & 1+\sum_{i=2}^{d_{1}+1} T_{n}\left(h^{\prime}-i+2, l^{\prime}-1, d\right) \\
& +\sum_{i=2}^{d_{2}+1} T_{n}\left(h^{\prime}-d_{2}+i-2, l^{\prime}-1, d\right) \\
= & 1+\sum_{i=0}^{d_{1}-1} T_{n}\left(h^{\prime}-i, l^{\prime}-1, d\right) \\
& +\sum_{i=1}^{d_{2}} T_{n}\left(h^{\prime}-i, l^{\prime}-1, d\right) \\
\leqslant & 1+\sum_{i=0}^{\min \left\{h^{\prime}, d / 2\right\}-1} T_{n}\left(h^{\prime}-i, l^{\prime}-1, d\right) \\
& +T_{n}\left(h^{\prime}, l^{\prime}, d\right) . \\
& \sum_{i=1}^{\min \left\{h^{\prime}-1,(d-1) / 2\right\}} T_{n}\left(h^{\prime}-i, l^{\prime}-1, d\right)
\end{aligned}
$$

A completely symmetric proof shows that $v-u \leqslant T_{n}\left(h^{\prime}, l^{\prime}, d\right)$ for every $\operatorname{VP}\langle u, v\rangle$ of $\Psi$ with $h(u)=h-h^{\prime}+1, h(v)=h-h^{\prime}$ and $l-l^{\prime}$ VPs over it.

Starting from lemma 5.5, in order to determine the largest chain admitting a canonic $\langle h, l, d\rangle$-layout, it is sufficient to estimate the number of nodes contained in the tree $\Gamma(h, l, d)$, that is, $\Gamma_{n}(h, l, d)$. 
Before solving the recurrence on $T_{n}(h, l, d)$ and consequently estimate $\Gamma_{n}(h, l, d)$, we recall that given $n+1$ positive integers $m, k_{1}, \ldots, k_{n}$, such that, $m=k_{1}+\cdots+k_{n}$, the multinomial coefficient $\left(\begin{array}{c}m \\ k_{1}, \ldots, k_{n}\end{array}\right)$ is defined as $m ! /\left(k_{1} !\right.$. $k_{2}$ ! $\cdots k_{n}$ !) (see, for instance, [17]).

Lemma 5.6. For every $h>0, l>0$ and $d>1$, if $d$ is even

$$
\begin{aligned}
& T_{n}(h, l, d) \\
& =\sum_{i=1}^{l} \sum_{j=0}^{h-1} \sum_{\substack{0 \leqslant k_{d / 2-1} \leqslant k_{d / 2-2} \leqslant \ldots \leqslant k_{2} \leqslant k_{1} \leqslant i \\
k_{1}+k_{2}+\cdots+k_{d / 2-1}=j}} 2^{k_{1}} \\
& \quad \times\left(\begin{array}{l}
i-k_{1}, k_{1}-k_{2}, \ldots, k_{d / 2-2}-k_{d / 2-1}, k_{d / 2-1} \\
i
\end{array}\right),
\end{aligned}
$$

while if $d$ is odd

$$
\begin{aligned}
& T_{n}(h, l, d) \\
& \begin{array}{l}
=\sum_{i=1}^{l} \sum_{j=0}^{h-1} \sum_{\substack{k_{(d-1) / 2} \leqslant k_{(d-1) / 2-1} \leqslant \cdots \leqslant k_{2} \leqslant k_{1} \leqslant i \\
k_{1}+k_{2}+\cdots+k_{(d-1) / 2}=j}} 2^{k_{1}-k_{(d-1) / 2}} \\
\times\left(\begin{array}{c}
i \\
i-k_{1}, k_{1}-k_{2}, \ldots, k_{(d-1) / 2-1}-k_{(d-1) / 2}, k_{(d-1) / 2}
\end{array}\right) .
\end{array}
\end{aligned}
$$

Proof. Let $M$ be the matrix defined as follows:

$$
M_{i, j}=\left\{\begin{array}{l}
1 \quad \text { if } i=0 \text { and } j=0, \\
0 \quad \text { if } i=0 \text { and } j>0, \\
\sum_{t=\max \{0, j-\lfloor d / 2\rfloor+1\}}^{j} M_{i-1, t} \\
+\sum_{t=\max \{1, j-\lfloor(d-1) / 2\rfloor\}}^{j} M_{i-1, t} \text { otherwise. }
\end{array}\right.
$$

Note that a generic element $M_{i, j}$ represents the number of subtrees $T(h-j, l-i, d)$ and $T^{\mathrm{r}}(h-j, l-i, d)$ that occur in $T(h, l, d)$ or analogously in the expansion of the recursive definition of $T(h, l, d)$ until obtaining only trees of load $l-i$. Moreover, by the recurrence of $T_{n}$, it results that $\sum_{i=1}^{l} \sum_{j=0}^{h-1} M_{i, j}$ is exactly the number of nodes in $T(h, l, d)$ minus one, that is the value $T_{n}(h, l, d)$.

In order to determine the sum of the first $h$ columns and the $l$ rows without the first of $M$, we observe that each row $i$ of $M$ corresponds to the coefficients of the $i$ th power of the polynomial $\left(\left(x^{\lfloor d / 2\rfloor-1}+x^{\lfloor d / 2\rfloor-2}+\cdots+x+1\right)+\left(x^{\lfloor(d-1) / 2\rfloor}+\right.\right.$ $\left.\left.x^{\lfloor(d-1) / 2\rfloor-1}+\cdots+x\right)\right)^{i}$. More precisely, a generic element $M_{i, j}$ is equal to the coefficient of $x^{j}$ in the expansion of the polynomial $\left(\left(x^{\lfloor d / 2\rfloor-1}+x^{\lfloor d / 2\rfloor-2}+\cdots+x+1\right)+\left(x^{\lfloor(d-1) / 2\rfloor}+\right.\right.$ $\left.\left.x^{\mathrm{L}(d-1) / 2\rfloor-1}+\cdots+x\right)\right)^{i}$.

If $d$ is even, by applying $d / 2-1$ times the well-known equality $(a+b)^{i}=\sum_{k=0}^{i}\left(\begin{array}{l}i \\ k\end{array}\right) a^{k} b^{i-k}$ to $\left(2 x^{d / 2-1}+2 x^{d / 2-2}+\right.$ $\left.\cdots+2 x^{2}+2 x+1\right)^{i}$ with $a=2 x^{d / 2-1}+2 x^{d / 2-2}+\cdots+2 x^{2}$ $+2 x$ and $b=1$ and iterating the same argument, we obtain

$$
\begin{aligned}
& \left(2 x^{d / 2-1}+2 x^{d / 2-2}+\cdots+2 x^{2}+2 x+1\right)^{i} \\
& =\sum_{k_{1}=0}^{i}\left(\begin{array}{c}
i \\
k_{1}
\end{array}\right)\left(2 x^{d / 2-1}+2 x^{d / 2-2}+\cdots+2 x^{2}+2 x\right)^{k_{1}} \\
& =\sum_{k_{1}=0}^{i} 2^{k_{1}}\left(\begin{array}{c}
i \\
k_{1}
\end{array}\right)\left(x^{d / 2-2}+x^{d / 2-3}+\cdots+x+1\right)^{k_{1}} x^{k_{1}} \\
& =\sum_{k_{1}=0}^{i} 2^{k_{1}}\left(\begin{array}{c}
i \\
k_{1}
\end{array}\right) \sum_{k_{2}=0}^{k_{1}}\left(\begin{array}{l}
k_{1} \\
k_{2}
\end{array}\right)\left(x^{d / 2-3}+x^{d / 2-4}+\cdots\right. \\
& +x+1)^{k_{2}} x^{k_{1}+k_{2}}=\cdots \\
& =\sum_{k_{1}=0}^{i} 2^{k_{1}}\left(\begin{array}{c}
i \\
k_{1}
\end{array}\right) \sum_{k_{2}=0}^{k_{1}}\left(\begin{array}{l}
k_{1} \\
k_{2}
\end{array}\right) \ldots \\
& \times \sum_{k_{d / 2-1}=0}^{k_{d / 2-2}}\left(\begin{array}{l}
k_{d / 2-2} \\
k_{d / 2-1}
\end{array}\right) x^{k_{1}+k_{2}+\cdots+k_{d / 2-1}} \\
& =\sum_{k_{1}=0}^{i} \sum_{k_{2}=0}^{k_{1}} \ldots \sum_{k_{d / 2-1}=0}^{k_{d / 2-2}} 2^{k_{1}}\left(\begin{array}{c}
i \\
k_{1}
\end{array}\right)\left(\begin{array}{l}
k_{1} \\
k_{2}
\end{array}\right) \cdots \\
& \times\left(\begin{array}{l}
k_{d / 2-2} \\
k_{d / 2-1}
\end{array}\right) x^{k_{1}+k_{2}+\cdots+k_{d / 2-1}},
\end{aligned}
$$

that can be rewritten as

$$
\begin{gathered}
\sum_{0 \leqslant k_{d / 2-1} \leqslant k_{d / 2-2} \leqslant \cdots \leqslant k_{2} \leqslant k_{1} \leqslant i} 2^{k_{1}} \\
\times\left(\begin{array}{c}
i \\
k_{1}
\end{array}\right)\left(\begin{array}{l}
k_{1} \\
k_{2}
\end{array}\right) \cdots\left(\begin{array}{c}
k_{d / 2-2} \\
k_{d / 2-1}
\end{array}\right) x^{k_{1}+k_{2}+\cdots+k_{d / 2-1}} \\
=\sum_{j=0}^{i(d / 2-1)} \sum_{\substack{0 \leqslant k_{d / 2-1} \leqslant k_{d / 2-2} \leqslant \cdots \leqslant k_{2} \leqslant k_{1} \leqslant i \\
k_{1}+k_{2}+\cdots+k_{d / 2}=j}} 2^{k_{1}} \\
\times\left(\begin{array}{c}
i \\
k_{1}
\end{array}\right)\left(\begin{array}{c}
k_{1} \\
k_{2}
\end{array}\right) \cdots\left(\begin{array}{c}
k_{d / 2-2} \\
k_{d / 2-1}
\end{array}\right) x^{j} .
\end{gathered}
$$

Therefore, recalling the definition of multinomial coefficient and that $M_{i, j}$ is the coefficient of $x^{j}$ in $\left(2 x^{d / 2-1}+2 x^{d / 2-2}+\right.$ $\left.\cdots+2 x^{2}+2 x+1\right)^{i}$, it follows that

$$
\begin{aligned}
M_{i, j}= & \sum_{\substack{0 \leqslant k_{d / 2-1} \leqslant k_{d / 2-2} \leqslant \cdots \leqslant k_{2} \leqslant k_{1} \leqslant i \\
k_{1}+k_{2}+\cdots+k_{d / 2-1}=j}} 2^{k_{1}} \\
& \times\left(\begin{array}{c}
i \\
i-k_{1}, k_{1}-k_{2}, \ldots, k_{d / 2-2}-k_{d / 2-1}, k_{d / 2-1}
\end{array}\right) .
\end{aligned}
$$

For the case of odd $d$ we obtain

$$
\begin{aligned}
& \left(x^{(d-1) / 2}+2 x^{(d-1) / 2-1}+\cdots+2 x^{2}+2 x+1\right)^{i} \\
& =\sum_{k_{1}=0}^{i}\left(\begin{array}{c}
i \\
k_{1}
\end{array}\right)\left(x^{(d-1) / 2}+2 x^{(d-1) / 2-1}+\cdots+2 x^{2}+2 x\right)^{k_{1}} \\
& =\sum_{k_{1}=0}^{i}\left(\begin{array}{c}
i \\
k_{1}
\end{array}\right)\left(x^{(d-1) / 2-1}\right. \\
& \left.\quad+2 x^{(d-1) / 2-2}+\cdots+2 x+2\right)^{k_{1}} x^{k_{1}}
\end{aligned}
$$




$$
\begin{aligned}
= & \sum_{k_{1}=0}^{i}\left(\begin{array}{c}
i \\
k_{1}
\end{array}\right) \sum_{k_{2}=0}^{k_{1}}\left(\begin{array}{l}
k_{1} \\
k_{2}
\end{array}\right)\left(x^{(d-1) / 2-2}+2 x^{(d-1) / 2-3}+\cdots\right. \\
& +2 x+1)^{k_{2}} 2^{k_{1}-k_{2}} x^{k_{1}+k_{2}} \\
= & \sum_{k_{1}=0}^{i}\left(\begin{array}{c}
i \\
k_{1}
\end{array}\right) \sum_{k_{2}=0}^{k_{1}}\left(\begin{array}{l}
k_{1} \\
k_{2}
\end{array}\right) \sum_{k_{3}=0}^{k 2}\left(\begin{array}{l}
k_{2} \\
k_{3}
\end{array}\right)\left(x^{(d-1) / 2-3}\right. \\
& \left.+2 x^{(d-1) / 2-4}+\cdots+2 x+1\right)^{k_{3}} 2^{k_{1}-k_{3}} x^{k_{1}+k_{2}+k_{3}} \\
= & \cdots \\
= & \sum_{k_{1}=0}^{i} \sum_{k_{2}=0}^{k_{1}} \sum_{k_{(d-1) / 2}=0}^{k_{(d-1) / 2-1}} 2^{k_{1}-k_{(d-1) / 2}}\left(\begin{array}{c}
i \\
k_{1}
\end{array}\right)\left(\begin{array}{l}
k_{1} \\
k_{2}
\end{array}\right) \cdots \\
& \times\left(\begin{array}{c}
k_{(d-1) / 2-1} \\
k_{(d-1) / 2}
\end{array}\right) x^{k_{1}+k_{2}+\cdots+k_{(d-1) / 2}},
\end{aligned}
$$

that can be rewritten as

$$
\begin{gathered}
\sum_{0 \leqslant k_{(d-1) / 2} \leqslant k_{(d-1) / 2-1} \leqslant \cdots \leqslant k_{2} \leqslant k_{1} \leqslant i} 2^{k_{1}-k_{(d-1) / 2}} \\
\times\left(\begin{array}{c}
i \\
k_{1}
\end{array}\right)\left(\begin{array}{c}
k_{1} \\
k_{2}
\end{array}\right) \cdots\left(\begin{array}{c}
k_{(d-1) / 2-1} \\
k_{(d-1) / 2}
\end{array}\right) x^{k_{1}+k_{2}+\cdots+k_{(d-1) / 2}} \\
=\sum_{j=0}^{i((d-1) / 2)} \sum_{\substack{0 \leqslant k_{(d-1) / 2} \leqslant k_{(d-1) / 2-1} \leqslant \cdots \leqslant k_{2} \leqslant k_{1} \leqslant i \\
k_{1}+k_{2}+\cdots+k_{(d-1) / 2}=j}} 2^{k_{1}-k_{(d-1) / 2}} \\
\times\left(\begin{array}{c}
i \\
k_{1}
\end{array}\right)\left(\begin{array}{c}
k_{1} \\
k_{2}
\end{array}\right) \cdots\left(\begin{array}{c}
k_{(d-1) / 2-1} \\
k_{(d-1) / 2}
\end{array}\right) x^{j} .
\end{gathered}
$$

Therefore,

$$
\begin{aligned}
& M_{i, j}=\sum_{\substack{0 \leqslant k_{(d-1) / 2} \leqslant k_{(d-1) / 2-1} \leqslant \cdots \leqslant k_{2} \leqslant k_{1} \leqslant i \\
k_{1}+k_{2}+\cdots+k_{(d-1) / 2}=j}} 2^{k_{1}-k_{(d-1) / 2}} \\
& \quad \times\left(\begin{array}{c}
i \\
i-k_{1}, k_{1}-k_{2}, \ldots, k_{(d-1) / 2-1}-k_{(d-1) / 2}, k_{(d-1) / 2}
\end{array}\right) .
\end{aligned}
$$

In every case, the claim follows by recalling that $T_{n}(h, l, d)=$ $\sum_{i=1}^{l} \sum_{j=0}^{h-1} M_{i, j}$.

Theorem 5.7. For every $h>0, l>0$ and $d>1$, the maximum number of nodes reachable in a chain network by a canonic $\langle h, l, d\rangle$-layout is

$$
\begin{aligned}
& \Gamma_{n}(h, l, d) \\
& =1+\sum_{k=1}^{h} T_{n}(k, l, d) \\
& =1+\sum_{k=1}^{h} \sum_{i=1}^{l} \sum_{j=0}^{h-1} \sum_{\substack{k_{d / 2-1} \leqslant k_{d / 2-2} \leqslant \cdots \leqslant k_{2} \leqslant k_{1} \leqslant i \\
k_{1}+k_{2}+\cdots+k_{d / 2-1}=j}} 2^{k_{1}} \\
& \times\left(\begin{array}{c}
i \\
i-k_{1}, k_{1}-k_{2}, \ldots, k_{d / 2-2}-k_{d / 2-1}, k_{d / 2-1}
\end{array}\right),
\end{aligned}
$$

if $d$ is even, and

$$
\begin{aligned}
& \Gamma_{n}(h, l, d) \\
& =1+\sum_{k=1}^{h} \sum_{i=1}^{l} \sum_{j=0}^{h-1} \sum_{\substack{0 \leqslant k_{(d-1) / 2} \leqslant k_{(d-1) / 2-1} \leqslant \cdots \leqslant k_{2} \leqslant k_{1} \leqslant i \\
k_{1}+k_{2}+\cdots+k_{(d-1) / 2}=j}} 2^{k_{1}-k_{(d-1) / 2}} \\
& \times\left(\begin{array}{c}
i \\
i-k_{1}, k_{1}-k_{2}, \ldots k_{(d-1) / 2-1}-k_{(d-1) / 2}, k_{(d-1) / 2}
\end{array}\right),
\end{aligned}
$$

if $d$ is odd.

Unfortunately, $\Gamma_{n}(h, l, d)$ in general cannot be expressed by means of a more compact closed formula. However, in some cases it can be significantly simplified. For instance,

- $d=2: \Gamma_{n}(h, l, 2)=h \cdot l+1$.

In fact, by the definition of the matrix $M$ in the proof of lemma 5.6, the only non null elements of $M$ belong to the first column and their value is always equal to one. Hence, the number of the nodes of every $T_{n}(k, l, 2)$ is $l$ and

$$
\Gamma_{n}(h, l, 2)=1+\sum_{k=1}^{h} T_{n}(k, l-1,2)=1+h \cdot l .
$$

This coincides with the result obtained in the previous section, and in fact $\Gamma(h, l, 2)$ coincides with the layout construction depicted in figure 1.

- $d \geqslant 2 h: \Gamma_{n}(h, l, d)=\sum_{i=0}^{\min \{h, l\}} 2^{i-1}\left(\begin{array}{l}h \\ i\end{array}\right)\left(\begin{array}{l}l \\ i\end{array}\right)+\frac{1}{2}$.

In fact, in this case, our model and constructions coincide with the ones in [9].

\section{Conclusion}

The main question left open in the paper is if the family of the canonic layouts contains optimal layouts for $d>2$. Even if not claimed explicitly, our constructions show that this is true for $d \leqslant 2$ and the previous results shown in the literature seem to confirm this conjecture.

Moreover, it would nice to extend our results to more general topologies and to the case in which the physical and adjacency graphs are not coincident.

Another worth investigating issue is the extension to other communication patterns like multicast and all-to-all.

Finally, it would be worth to investigate the approximability of the layout construction problem for $d>1$.

\section{References}

[1] S. Ahn, R.P. Tsang, S.R. Tong and D.H.C. Du, Virtual path layout design on ATM networks, in: Proc. of the 13th INFOCOM Conf. (1994) pp. 192-200.

[2] B.A. Akyol and D.C. Cox. Rerouting for handoff in a wireless ATM network, in: Proc. of the IEEE Internat. Conf. on Universal Personal Communications (1996).

[3] L. Becchetti, P. Bertolazzi, C. Gaibisso and G. Gambosi, On the design of efficient ATM schemes, in: Proc. of the SOFSEM Conf., Lecture Notes in Computer Science, Vol. 1338 (Springer, New York, 1997) pp. 375-382. 
[4] J. Burgin and D. Dorman, Broadband ISDN resource management: The role of virtual paths, IEEE Communicatons Magazine 29 (1991).

[5] M. Cheng, S. Rajagopalan, L. Chang, G. Pollini and M. Barton. PCS mobility support over fixed ATM networks, IEEE Communications Magazine 35 (1997) 82-92.

[6] C. Chrysostomou, A. Pitsillides and F. Pavlidou, A survey of wireless ATM handover iusses, in: Proc. of the Internat. Symposium of $3 G$ Infrastructure and Services, 3GIS, Vol. 2/3 (2001) pp. 34-39.

[7] I. Cidon, O. Gerstel and S. Zaks, A scalable approach to routing in ATM networks, in: Proc. of the 8th Internat. Workshop on Distributed Algorithms, eds. G. Tel and P.M.B. Vitányi, Terschelling, The Netherlands (October 1994), Lecture Notes in Computer Sience, Vol. 857 (Springer, New York, 1994) pp. 209-222; submitted for publication in IEEE/ACM Transactions on Networking.

[8] R. Cohen and A. Segall, Connection management and rerouting in ATM networks, in: Proc. of the 13th INFOCOM Conf. (1994) pp. 184 191.

[9] Y. Dinitz, M. Feighelstein and S. Zaks, On optimal graphs embedded into path and rings, with analysis using $l_{1}$-spheres, in: Proc. of the $23 r d$ Internat. Workshop on Graph-Theoretic Concepts in Computer Science $(W G)$, Lecture Notes in Computer Science, Vol. 1335 (Springer, New York, 1997) pp. 171-183.

[10] T. Eilam, M. Flammini and S. Zaks, A complete characterization of the path layout construction problem for ATM networks with given hop count and load, in: Proc. of the 24th Internat. Colloquium on Automata, Languages and Programming (ICALP), Lecture Notes in Computer Science, Vol. 1256 (Springer, New York, 1997) pp. 527-537.

[11] M. Flammini, G. Gambosi and A. Navarra, Wireless ATM layouts for chain networks, in: Proc. of the 17th Internat. Parallel and Distributed Processing Symposium (IPDPS), 3rd Workshop on Wireless, Mobile and Ad Hoc Networks (WMAN), IEEE Computer Society (2003) p. 220.1 .

[12] M. Flammini, A. Gasparini, G. Gambosi and A. Navarra, Dynamic layouts for wireless ATM, in: Proc. of the 9th Internat. Conf. on Parallel and Distributed Computing (Euro-Par), Lecture Notes in Computer Science, Vol. 2790 (Springer, New York, 2003) pp. 1056-1063.

[13] O. Gerstel, I. Cidon and S. Zaks, The layout of virtual paths in ATM networks, IEEE/ACM Transactions on Networking 4(6) (1996) 873-884.

[14] O. Gerstel, A. Wool and S. Zaks, Optimal layouts on a chain ATM network, in: 3rd Annual European Symposium on Algorithms (ESA), Corfu, Greece (September 1995), Lecture Notes in Computer Science, Vol. 979 (Springer, New York, 1995) pp. 508-522; to appear in Discrete Applied Mathematics.

[15] O. Gerstel and S. Zaks, The virtual path layout problem in fast networks, in: Proc. of the 13th ACM Symposium on Principles of Distributed Computing, Los Angeles, USA (August 1994) pp. 235-243.

[16] J.D. Gibson, The Mobile Communications Handbook, 2nd ed. (CRC Press/IEEE Press, 1999).

[17] R.L. Graham, D.E. Knuth and O. Patashnik, Concrete Mathematics (Addison-Wesley, Reading, MA, 1989).

[18] R. Händler and M.N. Huber, Integrated Broadband Networks: An Introduction to ATM-Based Networks (Addison-Wesley, Reading, MA, 1991).

[19] ITU recommendation, I series, Blue Book (November 1990).

[20] R.M. Karp, On the computational complexity of combinatorial problems, Networks 5 (1975) 45-68.

[21] E. Kranakis, D. Krizanc and A. Pelc, Hop-congestion tradeoffs in ATM networks, in: Proc. of the 9th IEEE Symposium on Parallel and Distributed Processing (1995) pp. 662-668.

[22] M. Mouly and M.B. Pautet, The GSM System for Mobile Communications (Cell \& Sys, 1993).
[23] G. Parry, Wireless ATM MAC protocols - a literature survey, WARP Project - URL, http://vera.ee.und.ac.za/coe/warp (1999).

[24] C. Partridge, Gigabit Networking (Addison-Wesley, Reading, MA, 1994).

[25] K.I. Sato, S. Ohta and I. Tokizawa, Broad-band ATM network architecture based on virtual paths, IEEE Transactions on Communications 38(8) (1990) 1212-1222.

[26] Y. Sato and K.I. Sato, Virtual path and link capacity design for ATM networks, IEEE Journal on Selected Areas in Communications 9 (1991)

[27] D. Sobirk and J.M. Karlsson, A survey of wireless ATM MAC protocols, in: Proc. of the Internat. Conf. on the Performance and Management of Complex Communication Networks (PMCCN) (Chapman \& Hall, London, 1997).

[28] A. Srinivasan, Improved approximations for edge-disjoint paths, unsplittable flow, and related routing problems, in: Proc. of the 38th Annual IEEE Symposium on Foundations of Computer Science (FOCS) (IEEE Computer Society Press, Los Alamitos, CA, 1997) pp. 416-425.

[29] L. Stacho and I. Vrto, Virtual path layouts for some bounded degree networks, in: Proc. of the 3rd Colloquium on Structural Information and Communication Complexity (SIROCCO) (Carleton Univ. Press, 1996) pp. 269-278

[30] S. Zaks, Path layouts in ATM networks, in: Proc. of the SOFSEM Conf., Lecture Notes in Computer Science, Vol. 1338 (Springer, New York, 1997) pp. 144-160.

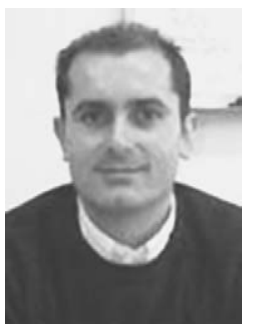

Michele Flammini received the degree in computer science at the University of L'Aquila in 1990 and the $\mathrm{Ph} . \mathrm{D}$. degree in computer science at the University of Rome "La Sapienza" in 1995. He is associate professor at the computer science Department of the University of L'Aquila since 2000. His research interests include algorithms and computational complexity, communication problems in interconnection networks and routing. He has authored and co-authored more than 50 papers in his fields of interest published in the most reputed international conferences and journals.

E-mail: flammini@di.univaq.it

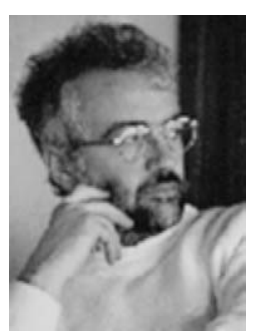

Giorgio Gambosi received the degree in electronic engineering at the University of Rome "La Sapienza" in 1980. He is full professor at the Department of Mathematics of the University of Rome "Tor Vergata". His research interests include distributed systems, network management algorithms and routing. He has coauthored about 70 research papers in relevant international conferences and journals.

E-mail: gambosi@mat.utovrm.it

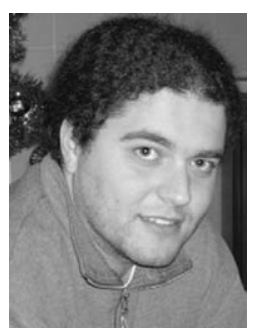

Alfredo Navarra received the degree in computer science at the University of L'Aquila in 2000. He spent one year at the research institute INRIA of Sophia Antipolis (France) collaborating with the MASCOTTE project group and now he is ending his $\mathrm{Ph} . \mathrm{D}$. degree in computer science at the University of Rome "La Sapienza". His research interests include algorithms and computational complexity, ATM, optical and wireless communication networks.

E-mail: navarra@di.univaq.it; navarra@sophia.inria.fr 